\title{
Nonverbal Teacher-student Communication in the Foreign Language Classroom
}

\author{
Qi Pan \\ English Department, Zhenjiang Watercraft College, Zhenjiang, China
}

\begin{abstract}
Nonverbal communication refers to a form of communication without using the words to repress oneself. Nonverbal communication is so basic that the teachers tend to take it for granted and always ignore it in the English classroom teaching. For attaining the goal of teaching, and improving teaching quality and efficiency in the foreign language classroom, the improvement of teaching method is a very important factor. Briefly introducing the definition and types of nonverbal communication, this paper discusses the functions and principles of using nonverbal communication in English teaching classroom and it explains some ways of using the nonverbal behaviors to improve the foreign language teaching. Therefore, the significance of nonverbal communication should be fully acknowledged by both teacher and students.
\end{abstract}

Index Terms - nonverbal communication, foreign language teaching

\section{INTRODUCTION}

For many years, researches of all backgrounds have been done in different aspects of communication. As we know, human beings communicate with others by exchanging information. Usually, there are two ways of exchanging information, one is verbal communication, and the other is nonverbal communication. On occasion, some research findings are concerned with verbal fashion, while some are from what is observed nonverbally. Nonverbal communication is only one facet of the giant field of communication, and yet it has been claimed that $65 \%$ of meaning is conveyed through nonverbal communication (Birdwhistell, 1970). One statement goes like this: we speak with our verbal organs while we converse with our bodies. Conservation not only makes up of a simple interchange of oral words As English teachers, our aim to teach language, especially a foreign language, is to teach the students how to use language to communicate more effectively with other people. However, we have laid much emphasis on the nonverbal communications, both in oral and written form, while neglecting that of nonverbal communication. Many teachers do not pay enough attention to nonverbal communication in class, contributing directly to failure in achieving teaching goals and students' acquisition of knowledge.

Studies on nonverbal communication have generally focused on the teaching methods in the classroom which may be used in teaching any subject. Learners haven't paid enough attention to the unique situation in language classes. Yet foreign language teachers who intentionally control their movements and position enjoy a delicate but effective power. During pattern drills, they can increase observably the tempo of the class, while decreasing teacher talk at the same time. Language teachers can use body language such as gestures to signal and instigate changes in the class atmosphere and thus further their instructional goals, throughout a class period encompassing such various activities as conversation and choral repetition. Furthermore, through nonverbal communication, they can effect personalization between class members and thus lessen the inhibitions which normally plague language students. It is suggested that a videotape recording of the class provides useful and objective feedback for teachers who wish to analyze and improve their own use of gesture and nonverbal communication in the classroom, Therefore, the significance of nonverbal communication should be fully acknowledged by both teacher and students. In fact, nonverbal communication plays an important role in our daily communication, and it is an indispensable part of language teaching. This paper will focus on the nonverbal communication taking place in the foreign language classroom.

\section{A. Concepts of Nonverbal Communication}

As we know, the communicative behaviors of human beings can be divided into two types: verbal communication and nonverbal communication. When it comes to the nonverbal communication, it has a rich history to some extent. The prime research of nonverbal communication can trace back to the Aristotle's research. Nonverbal communication refers to a form of communication without using the words to express oneself.

However, the Nonverbal communication in itself can be divided into many subcategories such as proxemics, kinesics, haptics and so on. (Samovar, 2000) Therefore, nonverbal communication is one academic research that has rich content and promote communication. In this paper, proxemics and kinesics will be discussed in particular, so literary review of them will be presented in great detail:

Proxemics refers to the use of space in a given situation. The use of space varies depending on the situation and the relation of the participants involved. Edward Hall has divided the use of space into 4 categories: intimate, personal, social, and public. (Edward Hall, 1966) An intimate interaction usually requires six to eighteen inches, personal 
interaction usually requires one and a half to three feet, social interaction requires three to five feet, and public interaction usually requires ten or more feet.

Kinesics is the term used for bodily movement. Body movement includes the use of eye contact, facial expressions, posture, and gesture. The use of bodily movement helps individuals express an emotional state, as well as the intensity of such a state. The second type of bodily movement is facial expression. An example of this would be the smile. A simple smile may carry with it many different meanings.

\section{B. Types of Nonverbal Communication}

Verbal communication only has oral form and written form. Different form verbal communication, nonverbal communication has many forms, including gesture, touch, body language, facial expression, eye contact and so on. According to the different standards of classifications, Bi Jiwan divided nonverbal communication into four types: body language, paralanguage, object communication, and physical environment. Body language refers to designation for instinctive, conscious and conventional expressive movements of the body, including posture, head movements, facial expressions and gesture and so on. Paralanguage, sometimes called vocalic, is the study of nonverbal cues of the voice. It includes voice quality, emotion, and speaking style, as well as prosodic features such as rhythm, intonation, and stress. These paralanguages may change the meaning of the words. Nonverbal communication can be communicated by object communication. And object communication includes clothing, hairstyle, architecture, symbols and so on. Physical environment also has influence on communication behaviors. However, this environment is not research about natural environment. It includes furniture, architectural style, interior decorating, lighting condition, colors, temperature, noise, and music. Also proxemics and chromatics also are physical environments. All physical environment factors may affect the behaviors of the communicators during interaction.

\section{Methodology}

As we know, The most appropriate methodology to use in this research study is a classroom observation schedule in which detailed notes and analysis are made based on nonverbal communication between teacher and students observed. I observed a first-year English class in my college. Every class consists of 80 minutes' classroom interaction between teacher and the students. The participants of this study included a teacher from my English Department and the students of Class 5 which consists of 25 male students.

The data was analyzed according to the aforementioned nonverbal communication categories: proxemics, kinesics, haptics and artifactual communication. However, due to time constraints I will focus on the first two categories-proxemics and kinesics-for this paper.

\section{Findings - Problems DURING The NONVERbal COMMUNiCATION BETWEEN TEACHER AND STUDENTS}

\section{A. Proxemics (Interpersonal Distance and Spatial Orientation)}

Proxemics refers to the use of space in a given situation. In classroom communication, the distance between teacher and students and physical classroom arrangement are the two major proxemics aspects.

a. Interpersonal distance

Interpersonal distance in the classroom, especially the distance between teacher and students, has profound influence on the effectiveness of teacher-pupil communication. However, nowadays not many teachers pay attention to this field of nonverbal communication. Some teachers just stand on the stage in front of the classroom during the whole process of a class and have little interaction with his students below.

b. Spatial orientation

The classroom itself has limited amount of space and the way that the kind of communication will certainly be affected. It is not only the seating arrangement of the classroom but also the way students are distributed in the class that affects significantly the students' learning. In the teacher-centered (traditional) seating arrangement style, students sit one after another in columns facing the teacher. The place they prefer to sit brings some advantages and disadvantages in terms of learning and participation. (Ikram, 2010)

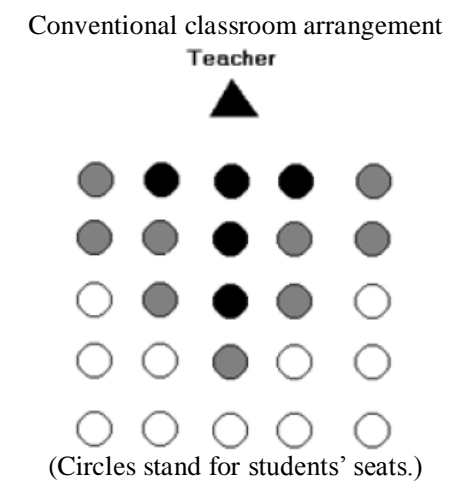


In this traditional type of classroom arrangement, people who seating the dark seats will bring about a majority of interaction between teacher and the students. People who occupying the gray positions account for some interaction, but less than those in the dark seats. However, students in the white circles will participate very infrequently. This kind of classroom arrangement causes physical and mental barrier between teacher and students.

\section{B. Posture Participance of Students}

The phenomenon that students who will not or cannot actively participate in classroom discussions is the most disappointing classroom phenomenon. Student withdrawal or fear of interacting not only deprives that student from sharing what he knows, but also deprives the teacher and other classmates from benefiting from what he can offer.

In the class discussion, the reasons why students lack of active participance are: (1) unconfidence: students who lack of confidence and regard themselves as incapable and tend to be silent out of shame; (2) different cutures: communicators are often affected by culture difference and nearly forbidden from speaking up, such as the deference out of respect for teachers' opinions, higher status students' or elder students ideas, and gender or race difference; (3) to prevent contradiction: shy, inexperient, or less unconfident individuals depend on keeping silent in preventing contradiction. Therefore, this strategy needs to be confronted delicately with alternative strategies offered as substitutes for unwanted silence, such avoidance should commonly be easy to diagnose by alert teachers.

\section{Direction and Movements of Gaze}

Teachers often forget about the importance of nonverbal communication in their own and their students' performance, although they know that speech is only one part of communication. Mentioned nonverbal communication, the eye contact is a useful tool for both the teacher and the student, however, much time is spent with eyes firmly fixed on the book, the blackboard, the window, the floor, or learning environment during the class. During class, when the teacher is looking at the students or seems to be paying attention to them, most of them tend to withdraw or move away their gazes which were previously on their teachers instinctively due to shyness.

\section{ReCOMmENDATIONS-How TO ENHANCE TEACHER-PUPIL NONVERBAL COMMUNiCATION}

\section{A. Improve Proxemics Communication}

It is essential that conflicts exist during the cross cultural communication. If we want to communicate with each other more smoothly, we must respect and learn the culture differences and the reasons behind them.

a. Interpersonal distance

When the teacher is giving a lecture, he should walk off the stage to join the crowd of students and make moderate interaction with them instead of standing on the stage all the time.

When a student is answering a question, the teacher can lean a little bit to him to listen attentively, showing interest and attention on the teacher's part.

b. Alternatives of traditional classroom arrangement

Kimberley Thoresen in his article physical classroom arrangement provides two ways of classroom arrangements. Each one has its own advantages based on the scale of the class and the teaching goal of the teacher.

(a). Horseshoe classroom arrangement

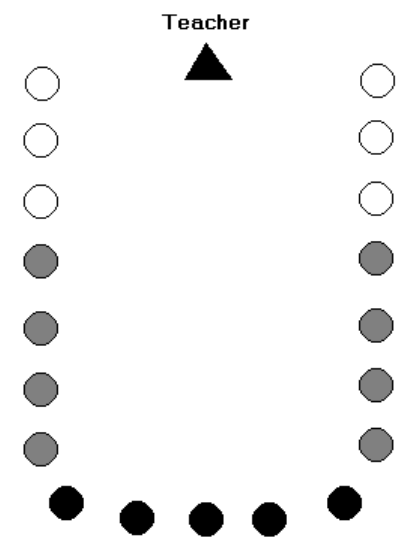

(Different colors of seats have same meaning as above)

Classes with quite small enrollments can be arranged in this fashion. Such an arrangement provides for each student equivalent visual access to most other students and the teacher.

Through this way, there is more participation in classes arranged. Students who are at the opposite end of the horseshoe from the teacher, however, are those most likely to interact, while those at the right and left hand of the teacher are those least likely to interact. (Kimberley, 2008) 
The horseshoe arrangement may be the most desirable, if the teacher hopes that the full-class interactions occur. That will encourage interaction both among the students and between students and teacher.

Moreover, this arrangement results wider participation than the traditional arrangement. It also seems reasonable to say that a teacher is perceived as less intimidating when he is seated in a circle with the students rather than behind the formal and imposing symbol of his large desk.

(b). Modular classroom arrangement

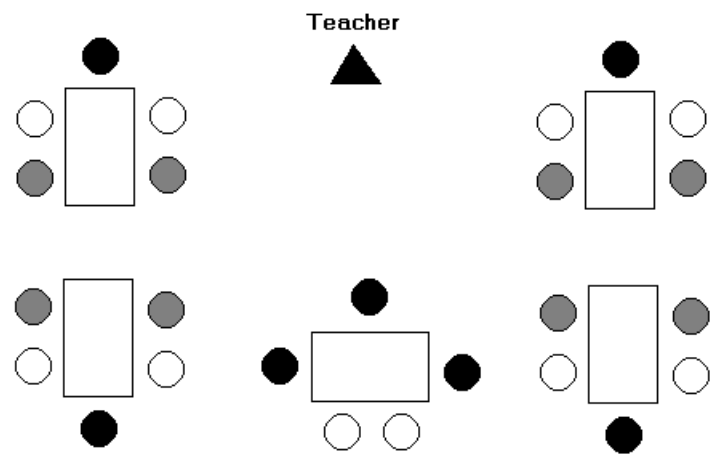

This arrangement is especially suitable for classes requiring interaction among smaller groups of students. Modular arrangements such as this one tend to increase the amount of student interaction.

The modular arrangement may be preferred, if quite important part of the learning in the class is rely upon student interaction with other students. This arrangement makes many students to be interacting at the same time without interrupting on one another. While many other elements will determine the nature of communication in a given teacher's classroom, the arrangements of classroom space may have the largest impact. (Kimberley, 2008)

\section{B. Break the Silence}

The solution lays mainly on the teacher's part, because he is the dominator of the classroom and he has certain authoritative power over students. The teacher needs to try to create learning and sharing atmosphere for the students, which will in return benefit both of the teacher and the students.

a. Offer a wide array of communication channels i.e.: discussion, debate, analysis, brainstorming, group work, etc.

Based on a case study of classroom management by Professor Fan Yi, he makes a case study of a double-period lesson of reading comprehension that he personally observed in Tiong Bahru Secondary School, Singapore, during his teaching practice. By recording some typical management problems in an English language class and by evaluating the strategies dealing with them, he gives supportive evidence to the thesis that the effective classroom management for an English language class is to create a positive class climate for learning.

With the aid of other approaches, communicative channels have been widely employed in the English language teaching in Singapore schools. Communicative channels are established on such a psycholinguistic assumption that effective language teaching and efficient language learning only occur in a positive class climate, which involves three essentials ---easy atmosphere, motivating environment and active participation (Widdowson, 1978; Littlewood, 1984).

The teacher had noticed that although the class discipline had been much enhanced, the stressful atmosphere and poor motivation hindered the pupils from participating in learning activities. In the second period of the lesson, she employed some remedial strategies.

First, in order to break the tense atmosphere in class and motivate the pupils for the topic, the teacher did not go straight to the second part of the essay. Instead she asked the class a few questions about their own personal eating experience, such as "Where do the Singaporeans go for meals?" "How many different styles of food can we eat in Singapore?" "Where did you have your Chinese New Year dinner?" and "What food did you have for the dinner?". These questions were very stimulating and immediately stirred up the class into a hot discussion. Some boys even stood up to offer their answers. A silent class now became very alive. When the students had been involved in the class discussion, the teacher turned the topic to the text. It was still group work, but each group member had to prepare for one of the given questions and present it to the group. After every group member presented it to the group, the group representative summed up their ideas and presented them to the class. This strategy involved every pupil in the learning activity. Each pupil had a clear task to fulfill and a strong desire to communicate with other group members. In order to make a successful presentation, he had to read the text and prepare for the given question carefully, integrating reading the text, writing down the notes, listening to the others and speaking to the group all together. Effective learning started from the moment the pupils took active part in learning activities. Some pupils had a tendency to speak dialect, but once he had got a role to play as the representative to speak up to the group or the class, he had to communicate in English. Thus dialect was prevented in the class. Obviously, by employing the remedial strategies, the teacher had rather 
successfully conducted the second period of the lesson. Although the remedial strategies were used to deal with specific management problems in a specific lesson, they were of general significance and applicability. Setting up an easy class atmosphere certainly helps to diminish the tension and anxiety existing in the class. Associating the lesson with the pupils' personal experience and interest always helps to motivate the pupils for the lesson. By giving pupils specific tasks, the teacher supplies them with desire for communication in English. All these strategies certainly involve pupils in learning activities.(Fan,2000)

b. Initially create "no lose" situations for students. Construct options where each is a winner to instill confidence and foster a greater willingness to participate.

A general knowledge contest was held in the College. There were all together 5 classes representing 5 groups in this contest, so it was like having a class but with larger scale. Eventually, each group was awarded some honor. Some particularly outstanding students in acting or grasp of profound knowledge were awarded special prizes. As a fact, our group did not do a good job in the contest, but we were still awarded the third prize. Though it was kind of consolation prize, it made us feel less embarrassed and feel willing to take part in this kind of activity again. At the same time, those special prizes profoundly showed our teachers' appreciation for the performance and participating of the students.

c. Be patient with reticent students. Do not rush them nor allow them to stall and thus have others called upon to fill the silence gap.

According to the classroom observation, 2 situations have great worth elaborating. When a reticent student was urged by the teacher to answer a certain question, she seemed quite anxious. Her face blushed and her murmuring demonstrated her blanc in mind. She tried great effort to find the answer from her book and from her classmates, while she gave up her own thinking for this question.

For another reticent student, the teacher posed a question to her. Before she got anxious about not being able to finding the answer, the teacher began talking about relevant details about this problem until she had her own opinion of this question. Afterwards, the teacher didn't judge whether it was right or wrong. Instead, she asked opinion of another student of this class, thus triggling a heated discussion on this issue among students.

Apparently, the second situation is more helpful for students' intellectual and mental growth. The teacher showed more understanding and respect for the student and she didn't let silence defeat this girl.

\section{Break the Shyness}

a. Motivating students with a smile

If the teacher wants to improve the students' performance, he can implement some simple and positive behaviors in his classroom and models these behaviors for his students.

A smile of teacher has great influence on students, especially those sentimental ones. The will feel warm-hearted, motivated, and more willing to acquire new knowledge.

b. Use eye contact proactively

Some teachers are good at dealing with interpersonal relationships, and they do well in gaining attention from the students from the beginning of the class. Their appealing sounds pleasant and makes people be willing to follow. The lack of eye contact is sometimes due to the absence of a clear appeal from the teacher.

Susan has done a case study of Ms. Rossi who has a classroom full of energetic juniors and she introduces to the reader how Ms. Rossi makes her students to pay full attention to her class. Ms. Rossi begins a lesson by saying, "I need all eyes on me." With continuous teacher-pupil eye contact, she gives her students an effective and informative class.

The importance of establishing and maintaining eye contact when we encourage students to make friendly and respectful eye contact with each other is greatly acknowledged. But do we pay attention to our own use of eye contact? Making eye contact with individual students can help a teacher establish a presence in the classroom and reinforces the importance of the teacher's message. (Hodge, 1971) It may also assist students in their ability to recall information. In one study, students whose teacher made eye contact with them while reading a story had greater recall of details of that story than students whose teacher did not make eye contact while reading the same story. (Otteson \& Otteson, 1980, as cited in Rosa, 2003) If the teacher decides that he'd like to improve his use of eye contact, he can enlist the children's help by letting them know that he'll be practicing his use of eye contact during lessons with them just as they practice good eye contact. Simply letting the children know that this is a goal he is working on can help keep them focused on the goal.

\section{CONCLUSION}

Human communication is divided into verbal communication and nonverbal communication, verbal communication is the most basic and most commonly used communication mode of human beings, and nonverbal communication is the essential part of human communication. In teaching Chinese as a foreign language, nonverbal communication also plays a very important role, in certain circumstances, non-verbal communication can achieve the goal that even verbal communication can't, application of nonverbal communication in teaching Chinese as a foreign language must be considered, different forms of nonverbal communicative behavior play a very important role on the teaching Chinese as a foreign language.

The effective way to achieve your students' attention in your classroom is nonverbal communication. Your students 
send out their own messages throughout your lecture and give you vital clues to understand whether you are reaching them or if you need to alter your teaching style. Your own nonverbal messages can inspire your students to participate in your lesson, an important part of your job as a teacher. Other nonverbal commands can help you get control of a disruptive student and ensure peace in your classroom.

The students' attention is easily attracted by the use of body language, and the body language can also help them deepen their impression and imagination. Appropriate body language can reflect the teachers' temperament and demeanor to strengthen their personal glamour. Through this way, the students' ability of English will be certainly and greatly improved. However, the teachers' body language often exists during the oral language. We need use the body language accurately during the class, or it will cause a bad influence on the English teaching course.

In a nutshell, nonverbal communication plays an important role in communicative behaviors. Content of the nonverbal communication is pretty rich and has various forms. English teaching also is communicative behavior, so that it is unavoidable that nonverbal communication has effect on language teaching. In reality, nonverbal communication can improve teaching effect. On the other hand, language teacher can make language teaching more vivid and visualization. And teacher can get a better teaching result with nonverbal communication more easily than without nonverbal communication. Moreover, we all know that nonverbal language is affected by culture and it is difficult for us to observe the diversity in the use and interpretation of nonverbal communication when we communicate with foreigners. The same nonverbal communication may be interpreted differently by people from different culture. And different nonverbal communication may also be used to convey the same idea or emotion. So that if teacher can teach nonverbal communication knowledge of English, students can understand English easily and improve their communicative abilities. In one word, nonverbal communication is beneficial for us to language teaching.

\section{REFERENCES}

[1] Birdwhistell. (1970). Kinesics and Context. Philadelphia: University of Pennsylvania Press.

[2] Fan, Y. (2000). Classroom management: a case study. College English Journal, Vol. 4. No.11:179-180.

[3] Hall, E.T. (1966). The Hidden Dimension. Garden city, NY: Doubleday.

[4] Hodge. (1971). Interpersonal Classroom Communication through Eye Contact. Theory into Practice, 10(1971): $264-267$.

[5] Ikram. (2010). Classroom Geography: Who Sit Where In The Traditional Classrooms. The Journal of International Social Research. Vol. 3. No.10:87-90.

[6] Kimberley, T. (2008). Physical Classroom Arrangement. EDUC 345 Assignment 2.

[7] Littlewood, W.T. (1984). Foreign and Second Language Learning. Oxford: Cambridge University Press.

[8] Otteson \&Otteson. (1980). Effects of Teacher Gaze on Children's Story. Perceptual and Motor Skills.

[9] Patrick. (1988). Nonverbal Communication. USA: Natl Eduaction Assen.

[10] Samovar. (2000). Communication Between Cultures. Beijing: Foreign Language Teaching and Research Press.

[11] Shen Minxian. (1999). The Use of the Body Language in Elementary School. Shanghai: Shanghai Education Vol.

[12] Susan B. Rosa. (2003). Using nonverbal communication to improve teacher effectiveness. Responsive Classroom Newsletter Fall. Vol. 15 No. 4.

[13] Widdowson, H.G. (1978). Teaching Language as Communication. Oxford: Oxford University Press.

Qi Pan was born in Zhenjiang, China in 1987. She received her B.A. degree in English language and literature from Jiangsu University of Science and Technology, China in 2009.

She is currently a tutor in English Department, Zhenjiang Watercraft College, Zhenjiang, China. Her research interests include Second Language Acquisition Contrastive Studies of English and Chinese. 